OPEN ACCESS

Edited by:

Jiyong Hong,

Duke University, United States

Reviewed by:

Xiao-Wei Liang

Duke University, United States Jongkook Lee

Kangwon National University,

South Korea

${ }^{*}$ Correspondence:

Changjin Lim

limcj@jbnu.ac.k

Young Taek Han

hanyt@dankook.ac.kr

Specialty section

This article was submitted to

Organic Chemistry,

a section of the journa

Frontiers in Chemistry

Received: 11 June 2020

Accepted: 24 July 2020

Published: 04 September 2020

Citation:

Yoon JA, Lim C and Han YT (2020)

Preliminary Study on Novel Expedient

Synthesis of 5-Azaisocoumarins by

Transition Metal-Catalyzed

Cycloisomerization

Front. Chem. 8:772

doi: 10.3389/fchem.2020.00772

\section{Preliminary Study on Novel Expedient Synthesis of 5-Azaisocoumarins by Transition Metal-Catalyzed Cycloisomerization}

\author{
Jeong A Yoon ${ }^{1}$, Changjin Lim ${ }^{2 *}$ and Young Taek Han ${ }^{1 *}$ \\ ${ }^{1}$ College of Pharmacy, Dankook University, Cheonan-si, South Korea, ${ }^{2}$ School of Pharmacy, Jeonbuk National University, \\ Jeonju-si, South Korea
}

A preliminary study to develop a novel synthetic method for 3-aryl-5-azaisocoumarins was performed herein. The cycloisomerization of $N$-pyranonyl propargylamines in the AgOTf-catalyzed system efficiently afforded the desired 3-aryl-5-azaisocoumarins in a highly regioselective manner. This unprecedented method is expected as an expedient alternative synthetic route to 5 -azaisocoumarins because the regioselectivity problem is circumvented, and it is easier to introduce substituents on the pyridine ring compared to previously reported intramolecular lactonization approaches.

Keywords: 5-azaisocoumarin, cycloisomerization, synthetic method, AgOTf, $\mathrm{N}$-pyranonyl propargylamine, Ag(I)-catalysis

\section{INTRODUCTION}

Isocoumarins, including 3,4-dihydroisocoumarin, are naturally abundant flavonoid scaffolds, that exhibit diverse pharmacological activities (Saeed, 2016; Saddiqa et al., 2017). In particular, 3-aryl isocoumarins possess unique and potent biological functions including antifungal (Saeed, 2003), anti-diabetic (Kim et al., 2017), immunomodulatory (Matsuda et al., 1998), antiallergic (Yoshikawa et al., 1992, 1994, 1996), and antimicrobial (Yoshikawa et al., 1992, 1994, 1996) activities, attracting the attention of synthetic and medicinal chemists. In medicinal chemistry, pyridine is considered to be the most efficient and popular bioisosteres with a phenyl moiety to improve biological activities and PK/PD profiles (Foye, 2008; Gaikwad et al., 2012). In this regard, azaisocoumarins, including 3-aryl-5-azaisocoumarin, are potential privileged scaffolds for developing drug candidates to treat a diverse range of diseases. Hence, there has been significant interest in the development of an efficient and novel synthetic route to 3-aryl-5-azaisocoumarin.

Despite the continual development of synthetic strategies for 3-aryl-isocoumarin analogs because of their interesting biological activities (Pal et al., 2011; Saddiqa et al., 2017), only a few methods and examples have been reported for the synthesis of 3-aryl-5-azaisocoumarin (Hellal et al., 2008; Li et al., 2010; Begouin and Maria-João, 2011; Panda et al., 2011; Park et al., 2011; Singh et al., 2018). To date, the synthesis of 3-aryl-5-azaisocoumarins has mostly been accomplished in the same manner as that of 3-aryl-isocoumarins, i.e., via construction of the 2-pyrone moiety in the final step via the intramolecular lactonization of 2-alkylnyl nicotinate derivatives. Because lactonization between the activated alkyne and ortho-carboxylate can occur by the 6-endo-dig or 5-exo-dig cyclization sequence (pathways a and b, respectively, in Figure 1), selectivity against methylenefuranone skeleton is a major challenge in these 2-pyrone formation routes. For instance, $\mathrm{Li}$ et al. reported the synthesis of 3-aryl-5-azaisocoumarin the 
Previous 2-pyrone formation approaches)

Examples

a)<smiles>O=Cc1cccnc1C#Cc1ccccc1</smiles>

b)

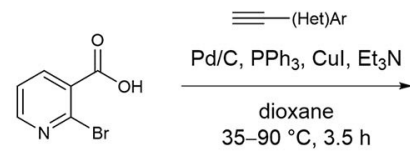

c)

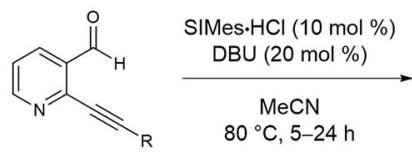

$\mathrm{NaClO}_{2}, \mathrm{NaH}_{2} \mathrm{PO}_{4}$

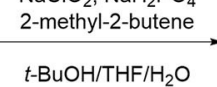

$0-50^{\circ} \mathrm{C}, 24 \mathrm{~h}$

$\equiv$ (Het)Ar

$35-90^{\circ} \mathrm{C}, 3.5 \mathrm{~h}$

$80^{\circ} \mathrm{C}, 5-24 \mathrm{~h}$

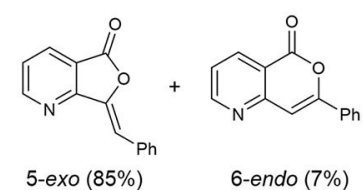

5-exo $(85 \%) \quad 6$-endo $(7 \%)$<smiles>O=C1O/C(=C\[AlH2])c2ncccc21</smiles>

5-exo (0-55\%)

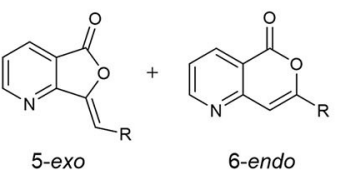

d)<smiles>CC(=O)c1cccnc1C#Cc1ccccc1</smiles>
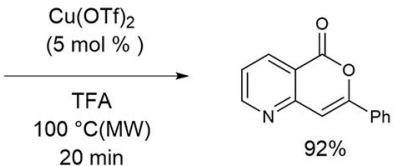

e)
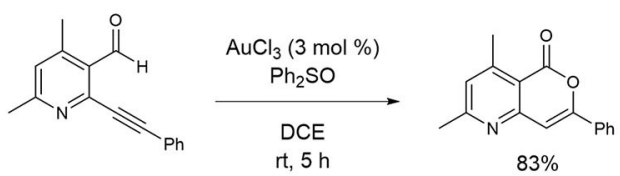

f)

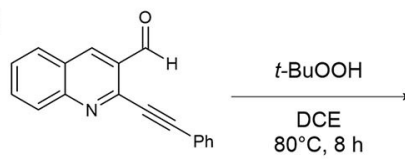

$$
\begin{array}{crr}
\mathrm{R} & 5 \text {-exo } & \text { 6-endo } \\
\hline n \text {-Bu } & 0 \% & 74 \% \\
\mathrm{Ph} & 41 \% & 33 \%
\end{array}
$$

This work)

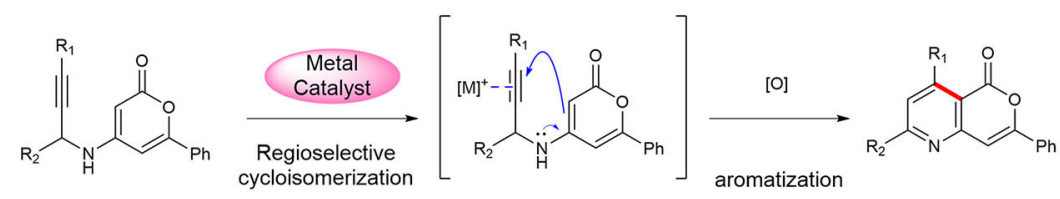

FIGURE 1 | Approaches for the synthesis of 5-azaisocoumarins. (a) Li's synthesis of 5-azaisocoumarins; (b) Queiroz's synthesis of 5-azaisocoumarins; (c) Youn's synthesis of 5-azaisocoumarins; (d) Bihel's synthesis of 5-azaisocoumarins; (e) Sarkar's synthesis of 5-azaisocoumarins; (f) Singh's synthesis of 5-azaisocoumarins.

Pinnick-type oxidative lactonization of 2-(phenylethynyl)nicotinaldehyde in 2010 (Figure 1a) (Li et al., 2010). The transition metal-mediated intramolecular lactonizations of 2-alkynyl nicotinates or 2-alkynyl nicotinaldehyde are popular approaches to obtain 5-azaisocoumarins by construction of the 2-pyrone moiety. Queiroz et al. attempted to make the 2-pyrone moiety of 3-aryl-5-azaisocoumarin via the one-pot Sonogashira coupling/transition metal catalyzed alkyne activation cascade (Figure 1b) (Begouin and Maria-João, 2011). These two methods produced 5-exo-cyclized furanone as a major product. Youn et al. adopted NHC-catalyzed alkyne activation for the synthesis of 5-azaisocoumarins, which selectively afforded a 6-endo or 5-exo cyclized product with appropriate reaction conditions (Figure 1c) (Park et al., 2011). 3-Butyl-5-azaisocoumarin was selectively synthesized via 6-endo cyclization mode from the corresponding precursor. However, for the arylethynyl precursor, the major product was the 5-exo-cyclized lactone instead of 3-aryl-5-azaisocoumarin. Several successful examples of 3-aryl-5-azaisocoumarin synthesis via selective formation of the 2-pyrone moiety have been reported. The Bihel (Figure 1d) (Hellal et al., 2008) and Sarkar groups (Figure 1e) (Panda et al., 2011) reported 3-aryl-5-azaisocoumarin synthesis by transition metal-catalyzed 6-endo-lactonization from ethynylnicotinate or ethynylnicotinaldehyde precursors, respectively. In addition, selective 6-endo-lactonization toward pyrano[4,3-b]quinolin-1-one, a phenyl-fused azaisocoumarin, was accomplished under metal/additive-free domino reaction conditions (Figure 1f) (Singh et al., 2018). In addition to the selectivity problem, previous 2-pyrone formation approaches are inefficient for the installation of substituents on pyridine moiety of 5-azaisocoumarins.

Recently, the metal-catalyzed 6-endo-dig cycloisomerization of $N$-propargyl enaminone derivatives has been widely used for the synthesis of pyridine-fused complex heterocycles, including azaanthraquinone, and steroidal pyridine (Abbiati et al., 2003; Yan et al., 2007; Jiang et al., 2010; Fei et al., 2011). In 
addition, intensive efforts to establish efficient synthetic methods for pyridine-fused coumarins and natural products using the metal-catalyzed cycloisomerization of $N$-propargyl enaminone intermediates have been reported (Ahn et al., 2019; Yoon and Han, 2019). In these studies, the pyridine moiety was constructed in a highly 6-endo-selective manner, which can be explained by $\sigma$-bond formation between the nucleophilic enolizable $\alpha$ position and exo-position of the electrophilic alkyne-metal complex and subsequent spontaneous oxidation to form a stable aromatic pyridine ring (Abbiati et al., 2003; Yan et al., 2007; Cacchi et al., 2008; Jiang et al., 2010; Fei et al., 2011; Mikušek et al., 2016; Lyubov'N et al., 2018; Ahn et al., 2019). It was anticipated that construction of the pyridine moiety via cycloisomerization of $\mathrm{N}$-propargylamine intermediates in the last-stage would be an efficient strategy for the synthesis of 3aryl-5-azaisocoumarins. As part of our effort to develop a novel synthetic approach toward privileged 3-aryl-5-azaisocoumarin scaffolds, we investigated the metal-catalyzed cycloisomerization of $N$-pyranonyl propargylamines.

Herein, we describe a concise synthesis of 3-aryl-5azaisocoumarins via AgOTf-catalyzed cycloisomerization, which to the best of our knowledge, has not been reported to date.

\section{MATERIALS AND METHODS}

\section{General Information}

Unless noted otherwise, all starting materials and reagents were obtained commercially and were used without further purification. All solvents utilized for routine product isolation and chromatography were of reagent grade and glass-distilled, and reaction flasks were dried at $100^{\circ} \mathrm{C}$ before use. Flash column chromatography was performed using silica gel 60 (230-400 mesh, Merck, Kenilworth, NJ, USA) with the indicated solvents. Thin-layer chromatography (TLC) was performed using 0.25mm silica gel plates (Merck, Kenilworth, NJ, USA). Mass spectra were obtained using an Agilent 6530 Q-TOF (Santa Clara, CA, USA) instrument. Infrared spectra were recorded on a JASCO FT-IR-4200 spectrometer (Tokyo, Japan). ${ }^{1} \mathrm{H}$ and ${ }^{13} \mathrm{C}$ spectra were recorded on a Brucker Analytik ADVANCE digital 500 (500 MHz) (Billerica, MA, USA) and BRUKER AVANCE-800 (Billerica, MA, USA). Chemical shifts are expressed in parts per million (ppm, $\delta$ ) downfield from tetramethylsilane and are referenced to the deuterated solvent. ${ }^{1} \mathrm{HNMR}$ data are reported in the order of chemical shift, multiplicity (s, singlet; $d$, doublet; t, triplet; q, quartet; m, multiplet; bs, broad singlet; and/or multiple resonances), number of protons, and coupling constant in hertz $(\mathrm{Hz})$.

\section{2-Oxo-6-phenyl-2H-pyran-4-yl 4-methylbenzenesulfonate (2)}

To a stirred solution of 1 (500 mg, $2.66 \mathrm{mmol})$ in $\mathrm{CH}_{2} \mathrm{Cl}_{2}$ $(20 \mathrm{~mL})$ were added tosyl chloride $(507 \mathrm{mg}, 2.66 \mathrm{mmol})$ and $\mathrm{Et}_{3} \mathrm{~N}$ $(1.11 \mathrm{~mL}, 7.98 \mathrm{mmol})$ at ambient temperature. After stirring for $3 \mathrm{~h}$, the reaction mixture was quenched with water and extracted with $\mathrm{CH}_{2} \mathrm{Cl}_{2}$. The combined organic layer was washed with brine, dried over $\mathrm{MgSO}_{4}$, and concentrated in vacuo. The residue was purified by flash column chromatography (EtOAc/ $n$-Hexane $=1: 5)$ to give $838 \mathrm{mg}(92 \%)$ of $\mathbf{2}$ as a yellow solid.

m.p.: $128-130^{\circ} \mathrm{C} ; \mathrm{R}_{\mathrm{f}}=0.67($ EtOAc $/ n$-Hexane $=1: 1) ;{ }^{1} \mathrm{H}$ NMR $\left(500 \mathrm{MHz}, \mathrm{CDCl}_{3}\right) \delta 7.86(\mathrm{~d}, 2 \mathrm{H}, J=8.3 \mathrm{~Hz}, \mathrm{Ar}-\mathrm{H}), 7.78$ (d, $2 \mathrm{H}, J=6.9 \mathrm{~Hz}, \mathrm{Ar}-\mathrm{H}), 7.51-7.45(\mathrm{~m}, 3 \mathrm{H}, \mathrm{Ar}-\mathrm{H}), 7.40$ (d, $2 \mathrm{H}$, $J=8.0 \mathrm{~Hz}, \mathrm{Ar}-\mathrm{H}), 6.61(\mathrm{~d}, 1 \mathrm{H}, J=1.8 \mathrm{~Hz}$, vinyl $\mathrm{H}), 5.91(\mathrm{~d}$, $1 \mathrm{H}, J=1.8 \mathrm{~Hz}$, cyclic-CH), $2.48\left(\mathrm{~s}, 3 \mathrm{H}, \mathrm{CH}_{3}\right) ;{ }^{13} \mathrm{C}-\mathrm{NMR}(125$ $\left.\mathrm{MHz}, \mathrm{CDCl}_{3}\right) \delta 162.4,162.3,162.2,146.9,131.9,131.8,130.5$, $130.5,129.2,128.6,126.0,101.7,98.3,22.0$; IR (thin film, neat) $v_{\max } 1,736,1,631,1,556,1,496,1,386,1,198,1,139,1,091,916,748$ $\mathrm{cm}^{-1}$; LR-MS (ESI+) $\mathrm{m} / z 343\left(\mathrm{M}+\mathrm{H}^{+}\right)$; HR-MS (ESI+) calcd for $\mathrm{C}_{18} \mathrm{H}_{15} \mathrm{O}_{5} \mathrm{~S}\left(\mathrm{M}+\mathrm{H}^{+}\right)$343.0635; found 343.0631 .

\section{General Procedure I for Nucleophilic Aromatic Substitution}

To a stirred solution of 2 (1 equiv.) and $N, N$ diisopropylethylamine (3 equiv.) in $\mathrm{MeCN}$ ( $0.1 \mathrm{M}$ solution) was added propargylamine hydrochloride ( 1.5 equiv.) at ambient temperature. After stirring for $14 \mathrm{~h}$ at $80^{\circ} \mathrm{C}$, the reaction mixture was quenched with $1 N \mathrm{HCl}$ and extracted with EtOAc. The combined organic layer was washed with brine, dried over $\mathrm{MgSO}_{4}$, and concentrated in vacuo. The residue was purified by flash column chromatography (EtOAc $/ n$-Hexane $=1: 1)$ to give title compounds (3a-3i).

\section{6-Phenyl-4-(prop-2-yn-1-ylamino)-3,4-dihydro-2H- pyran-2-one (3a)}

Yellow solid of $3 \mathrm{a}(1.13 \mathrm{~g}, 69 \%)$ was obtained via General Procedure I from $2.50 \mathrm{~g}$ of 2 .

m.p.: $169-171^{\circ} \mathrm{C} ; \mathrm{R}_{\mathrm{f}}=0.29($ EtOAc/n-Hexane $=1: 1) ;{ }^{1} \mathrm{H}$ NMR (500 MHz, DMSO-d 6 ) $\delta 7.72(\mathrm{~s}, 3 \mathrm{H}, \mathrm{Ar}-\mathrm{H}), 7.54-7.51(\mathrm{~m}$, $2 \mathrm{H}, \mathrm{Ar}-\mathrm{H}), 6.55$ (s, $1 \mathrm{H}$, vinyl $\mathrm{H}), 5.03(\mathrm{~d}, 1 \mathrm{H}, J=1.3 \mathrm{~Hz}$, vinyl $\mathrm{H})$, $4.01\left(\mathrm{~s}, 2 \mathrm{H}, \mathrm{CH}_{2}\right), 3.30(\mathrm{t}, 1 \mathrm{H}, J=2.3 \mathrm{~Hz}, \mathrm{CH}) ;{ }^{13} \mathrm{C}-\mathrm{NMR}(125$ MHz, DMSO-d 6 ) $\delta 162.5,157.8,157.1,131.6,130.6,129.1,125.1$, 97.0, 80.7, 79.5, 74.6, 31.3; IR (thin film, neat) $v_{\max } 3,289,3,089$, $1,666,1,549,1,298,1,198,1,063,847,767,691 \mathrm{~cm}^{-1}$; LR-MS $(\mathrm{ESI}+) \mathrm{m} / z 226\left(\mathrm{M}+\mathrm{H}^{+}\right)$; HR-MS (ESI+) calcd for $\mathrm{C}_{14} \mathrm{H}_{12} \mathrm{NO}_{2}$ $\left(\mathrm{M}+\mathrm{H}^{+}\right)$226.0863; found 226.0856 .

\section{4-(But-2-yn-1-ylamino)-6-phenyl-3,4-dihydro-2H- pyran-2-one (3b)}

Yellow solid of $\mathbf{3 b}(25 \mathrm{mg}, 72 \%)$ was obtained via General Procedure I from $50 \mathrm{mg}$ of $\mathbf{2}$.

m.p.: $181-183^{\circ} \mathrm{C} ; \mathrm{R}_{\mathrm{f}}=0.38($ EtOAc/n-Hexane $=1: 1) ;{ }^{1} \mathrm{H}$ NMR (500 MHz, DMSO-d 6 ) $\delta 7.72(\mathrm{~s}, 2 \mathrm{H}, \mathrm{Ar}-\mathrm{H}), 7.68(\mathrm{t}, 1 \mathrm{H}$, $J=5.3 \mathrm{~Hz}, \mathrm{NH}), 7.54-7.49(\mathrm{~m}, 3 \mathrm{H}, \mathrm{Ar}-\mathrm{H}), 6.54$ (s, $1 \mathrm{H}$, vinyl $\mathrm{H}), 4.99(\mathrm{~s}, 1 \mathrm{H}$, vinyl $\mathrm{H}), 3.94\left(\mathrm{~s}, 2 \mathrm{H}, \mathrm{CH}_{2}\right), 1.81(\mathrm{t}, 3 \mathrm{H}, J=$ $\left.2.1 \mathrm{~Hz}, \mathrm{CH}_{3}\right) ;{ }^{13} \mathrm{C}-\mathrm{NMR}\left(125 \mathrm{MHz}, \mathrm{DMSO}-\mathrm{d}_{6}\right) \delta 162.5,157.7$, 157.2, 131.6, 130.5, 129.1, 125.1, 97.0, 80.4, 79.6, 74.6, 31.6, 3.0; IR (thin film, neat) $v_{\max } 3,269,3,090,1,658,1,547,1,298,1,194$, 1,063, 992, 850, 768, $692 \mathrm{~cm}^{-1}$; LR-MS (ESI+) $\mathrm{m} / z 240(\mathrm{M}+$ $\left.\mathrm{H}^{+}\right)$; HR-MS (ESI+) calcd for $\mathrm{C}_{15} \mathrm{H}_{14} \mathrm{NO}_{2}\left(\mathrm{M}+\mathrm{H}^{+}\right)$240.1019; found 240.1018 . 


\section{4-(But-3-yn-2-ylamino)-6-phenyl-3,4-dihydro-2H- pyran-2-one (3c)}

Yellow solid of $3 \mathrm{c}(17 \mathrm{mg}, 48 \%)$ was obtained via General Procedure I from $50 \mathrm{mg}$ of 2.

m.p.: $190-191^{\circ} \mathrm{C} ; \mathrm{R}_{\mathrm{f}}=0.30($ EtOAc/n-Hexane $=1: 1) ;{ }^{1} \mathrm{H}$ NMR (500 MHz, DMSO-d 6 ) $\delta 7.72(\mathrm{~s}, 2 \mathrm{H}, \mathrm{Ar}-\mathrm{H}), 7.66(\mathrm{~d}, 1 \mathrm{H}$, $J=6.9 \mathrm{~Hz}, \mathrm{NH}), 7.54-7.50(\mathrm{~m}, 3 \mathrm{H}, \mathrm{Ar}-\mathrm{H}), 6.53(\mathrm{~s}, 1 \mathrm{H}$, vinyl $\mathrm{H}), 5.06(\mathrm{~d}, 1 \mathrm{H}, J=1.5 \mathrm{~Hz}$, vinyl $\mathrm{H}), 4.37(\mathrm{~s}, 1 \mathrm{H}, \mathrm{CH}), 3.32(\mathrm{~d}$, $1 \mathrm{H}, J=2.1 \mathrm{~Hz}, \mathrm{CH}), 1.43\left(\mathrm{~d}, 3 \mathrm{H}, J=6.8 \mathrm{~Hz}, \mathrm{CH}_{3}\right) ;{ }^{13} \mathrm{C}-\mathrm{NMR}$ $\left(125 \mathrm{MHz}, \mathrm{DMSO}-\mathrm{d}_{6}\right) \delta 162.5,156.9,156.4,131.6,130.5,129.1$, $126.9,125.1,97.0,83.8,81.0,73.5,20.9$; IR (thin film, neat) $v_{\max }$ 3,283, 3,066, 1,667, 1,550, 1,298, 1,198, 1,176, 1,063, 847, 758, 691 $\mathrm{cm}^{-1}$; LR-MS (ESI+) $m / z 240\left(\mathrm{M}+\mathrm{H}^{+}\right)$; HR-MS (ESI+) calcd for $\mathrm{C}_{15} \mathrm{H}_{14} \mathrm{NO}_{2}\left(\mathrm{M}+\mathrm{H}^{+}\right)$240.1019; found 240.1029.

\section{6-Phenyl-4-((3-phenylprop-2-yn-1-yl)amino)-3,4- dihydro-2H-pyran-2-one (3d)}

Yellow solid of $3 \mathbf{d}(24 \mathrm{mg}, 52 \%)$ was obtained via General Procedure I from $50 \mathrm{mg}$ of 2.

m.p.: $194-196^{\circ} \mathrm{C} ; \mathrm{R}_{\mathrm{f}}=0.24($ EtOAc/n-Hexane $=1: 1) ;{ }^{1} \mathrm{H}-$ NMR $\left(500 \mathrm{MHz}, \mathrm{DMSO}-\mathrm{d}_{6}\right), \delta 7.83(\mathrm{t}, 1 \mathrm{H}, J=5.3 \mathrm{~Hz}, \mathrm{Ar}-\mathrm{H})$, 7.74 (s, 1H, Ar-H), 7.54-7.51 (m, 3H, Ar-H), 7.45-7.38 (m, 5H, Ar-H), $6.59(\mathrm{~d}, 1 \mathrm{H}, J=6.6 \mathrm{~Hz}$, vinyl $\mathrm{H}), 5.12(\mathrm{~d}, 1 \mathrm{H}, J=1.3 \mathrm{~Hz}$, vinyl $\mathrm{H}$ ), $4.28\left(\mathrm{~s}, 2 \mathrm{H}, \mathrm{CH}_{2}\right) ;{ }^{13} \mathrm{C}-\mathrm{NMR}\left(125 \mathrm{MHz}, \mathrm{DMSO}-\mathrm{d}_{6}\right) \delta$ $162.5,157.9,157.2,131.6,131.4,130.6,129.1,128.8,128.7,125.1$, $121.9,97.1,85.4,83.1,80.7,32.1$; IR (thin film, neat) $v_{\max } 3,271$, 3,089, 1,663, 1,544, 1,451, 1,293, 1,199, 1,072, 849, 768, $691 \mathrm{~cm}^{-1}$; LR-MS (ESI+) $m / z 324\left(\mathrm{M}+\mathrm{Na}^{+}\right)$; HR-MS (ESI+) calcd for $\mathrm{C}_{20} \mathrm{H}_{15} \mathrm{NNaO}_{2}\left(\mathrm{M}+\mathrm{Na}^{+}\right)$324.0995; found 324.0990.

\section{6-phenyl-4-((3-(o-tolyl)prop-2-yn-1-yl)amino)-2H- pyran-2-one (3e)}

Yellow solid of 3 e $(69 \mathrm{mg}, 75 \%)$ was obtained via General Procedure I from $100 \mathrm{mg}$ of 2 .

m.p.: $163-164^{\circ} \mathrm{C} ; \mathrm{R}_{\mathrm{f}}=0.40($ EtOAc/n-Hexane $=1: 1) ;{ }^{1} \mathrm{H}-$ NMR (800 MHz, DMSO-d 6 ) $\delta 7.84(\mathrm{~s}, 1 \mathrm{H}, \mathrm{NH}), 7.74(\mathrm{~s}, 2 \mathrm{H}$, Ar-H), 7.55-7.48 (m, 3H, Ar-H), 7.38 (d, J = 7.6 Hz, 1H, Ar-H), 7.30-7.23 (m, 2H, Ar-H), 7.22 - 7.12 (m, 1H, Ar-H), 6.59 (s, 1H, vinyl $\mathrm{H}), 5.15(\mathrm{~d}, J=1.2 \mathrm{~Hz}, 1 \mathrm{H}$, vinyl $\mathrm{H}), 4.31\left(\mathrm{~s}, 2 \mathrm{H}, \mathrm{CH}_{2}\right), 2.36$ $\left(\mathrm{s}, 3 \mathrm{H}, \mathrm{CH}_{3}\right) ;{ }^{13} \mathrm{C}-\mathrm{NMR}\left(200 \mathrm{MHz}, \mathrm{DMSO}-\mathrm{d}_{6}\right) \delta 162.5,157.8$, 157.1, 139.7, 131.6, 131.6, 130.6, 129.6, 129.1, 128.7, 125.9, 125.1, $121.8,97.1,89.2,82.0,80.9,32.2,20.2$; IR (thin film, neat) $v_{\max }$ $3,263,3,072,1,664,1,544,1,289,1,182,1,027,759,689 \mathrm{~cm}^{-1}$; LR-MS (ESI+) $m / z 316\left(\mathrm{M}+\mathrm{H}^{+}\right)$; HR-MS (ESI+) calcd for $\mathrm{C}_{21} \mathrm{H}_{18} \mathrm{NO}_{2}\left(\mathrm{M}+\mathrm{H}^{+}\right)$316.1332; found 316.1333.

\section{4-(hex-5-en-2-yn-1-ylamino)-6-phenyl-2H-pyran-2- one $(3 \mathrm{~g})$}

Yellow solid of $\mathbf{3 g}$ (53 mg, 68\%) was obtained via General Procedure I from $100 \mathrm{mg}$ of 2 .

m.p.: $119-120^{\circ} \mathrm{C} ; \mathrm{R}_{\mathrm{f}}=0.39($ EtOAc/n-Hexane $=1: 1) ;{ }^{1} \mathrm{H}-$ NMR (800 MHz, DMSO-d 6 ) $\delta 7.82-7.65$ (m, 3H, Ar-H \& NH), 7.54-7.48 (m, 3H, Ar-H), 6.55 (s, 1H, vinyl H), 5.87-5.69 (ddt, $J$ $=17.0,10.1,5.2 \mathrm{~Hz}, 1 \mathrm{H}$, vinyl $\mathrm{H}), 5.30(\mathrm{dd}, J=17.0,1.7 \mathrm{~Hz}, 1 \mathrm{H}$, vinyl $\mathrm{H}), 5.10(\mathrm{~d}, J=9.7 \mathrm{~Hz}, 1 \mathrm{H}$, vinyl $\mathrm{H}), 5.03(\mathrm{~d}, J=1.5 \mathrm{~Hz}, 1 \mathrm{H}$, vinyl $\mathrm{H}), 4.02\left(\mathrm{~s}, 2 \mathrm{H}, \mathrm{CH}_{2}\right), 3.05-3.01\left(\mathrm{~m}, 2 \mathrm{H}, \mathrm{CH}_{2}\right) ;{ }^{13} \mathrm{C}-\mathrm{NMR}$ $\left(200 \mathrm{MHz}, \mathrm{DMSO}-\mathrm{d}_{6}\right) \delta 162.6,157.7,157.1,132.8,131.6,130.6$,
129.1, 125.1, 116.0, 97.0, 80.5, 80.3, 78.1, 31.7, 22.3; IR (thin film, neat) $v_{\max } 3,272,3,092,1,664,1,548,1,297,1,182,1,038,918,767$, $691 \mathrm{~cm}^{-1}$; LR-MS (ESI+) m/z $266\left(\mathrm{M}+\mathrm{H}^{+}\right)$; HR-MS (ESI+) calcd for $\mathrm{C}_{17} \mathrm{H}_{16} \mathrm{NO}_{2}\left(\mathrm{M}+\mathrm{H}^{+}\right)$266.1176; found 266.1169.

\section{4-((3-(cyclohex-2-en-1-yl)prop-2-yn-1-yl)amino)-6- phenyl-2H-pyran-2-one (3h)}

Yellow oil of $3 \mathbf{h}$ (39 mg, 44\%) was obtained via General Procedure I from $100 \mathrm{mg}$ of 2.

$\mathrm{R}_{\mathrm{f}}=0.35($ EtOAc $/ n$-Hexane $=1: 1) ;{ }^{1} \mathrm{H}-\mathrm{NMR}(800 \mathrm{MHz}$, DMSO-d 6 ) $\delta 7.72$ (s, 2H, Ar-H), 7.66 (s, 1H, NH), 7.54-7.48 (m, 3H, Ar-H), 6.55 (s, 1H, vinyl $\mathrm{H}), 5.72-5.68$ (m, 1H, vinyl $\mathrm{H}), 5.58-5.55(\mathrm{~m}, 1 \mathrm{H}$, vinyl $\mathrm{H}), 5.00(\mathrm{~s}, 1 \mathrm{H}$, vinyl $\mathrm{H}), 3.98(\mathrm{~s}$, $\left.2 \mathrm{H}, \mathrm{CH}_{2}\right), 3.18-3.11(\mathrm{~m}, 1 \mathrm{H}, \mathrm{CH}), 1.98-1.91\left(\mathrm{~m}, 2 \mathrm{H}, 2 \times \mathrm{CH}_{2}\right)$, 1.85-1.79 (m, $\left.1 \mathrm{H}, 1 / 2 \times \mathrm{CH}_{2}\right), 1.73-1.67\left(\mathrm{~m}, 1 \mathrm{H}, 1 / 2 \times \mathrm{CH}_{2}\right)$, 1.60-1.55 (m, $\left.1 \mathrm{H}, 1 / 2 \times \mathrm{CH}_{2}\right), 1.54-1.48\left(\mathrm{~m}, 1 \mathrm{H}, 1 / 2 \times \mathrm{CH}_{2}\right)$; ${ }^{13} \mathrm{C}-\mathrm{NMR}\left(200 \mathrm{MHz}, \mathrm{DMSO}-\mathrm{d}_{6}\right) \delta 162.5,157.7,157.1,131.6$, $130.5,129.1,127.8,126.8,125.1,97.1,86.4,80.4,75.3,31.7,28.9$, 26.6, 24.1, 20.0; IR (thin film, neat) $v_{\max } 3,117,2,887,1,670$, 1,564, 1,337, 1,245, 767, $688 \mathrm{~cm}^{-1}$; LR-MS (ESI+) $\mathrm{m} / z 306(\mathrm{M}+$ $\left.\mathrm{H}^{+}\right)$; HR-MS (ESI+) calcd for $\mathrm{C}_{20} \mathrm{H}_{20} \mathrm{NO}_{2}\left(\mathrm{M}+\mathrm{H}^{+}\right)$306.1489; found 306.1486.

\section{6-phenyl-4-((3-(triisopropylsilyl)prop-2-yn-1- yl)amino)-2H-pyran-2-one (3i)}

Yellow solid of $3 \mathbf{i}(80 \mathrm{mg}, 72 \%)$ was obtained via General Procedure I from $100 \mathrm{mg}$ of 2.

m.p.: $175-176^{\circ} \mathrm{C} ; \mathrm{R}_{\mathrm{f}}=0.60($ EtOAc $/ n$-Hexane $=1: 1) ;{ }^{1} \mathrm{H}-$ NMR (800 MHz, DMSO-d $\left.{ }_{6}\right) \delta 7.74$ (s, 2H, Ar-H), 7.68 (s, 1H, $\mathrm{NH}), 7.54-7.47$ (m, 3H, Ar-H), 6.57 (s, 1H, vinyl H), 5.07 (d, J $=1.9 \mathrm{~Hz}, 1 \mathrm{H}$, vinyl $\mathrm{H}), 4.09\left(\mathrm{~s}, 2 \mathrm{H}, \mathrm{CH}_{2}\right), 1.05-0.98(\mathrm{~m}, 21 \mathrm{H}$, TIPS-H); ${ }^{13} \mathrm{C}-\mathrm{NMR}\left(200 \mathrm{MHz}, \mathrm{DMSO}-\mathrm{d}_{6}\right) \delta 162.4,157.7,157.0$, 131.6, 130.5, 129.1, 125.1, 103.6, 97.0, 83.6, 81.1, 32.3, 18.4, 10.6; IR (thin film, neat) $v_{\max } 3,272,3,075,1,670,1,549,1,245,1,182$, 1,038, 883, 767, $677 \mathrm{~cm}^{-1}$; LR-MS (ESI+) $\mathrm{m} / z 382\left(\mathrm{M}+\mathrm{H}^{+}\right)$; HR-MS (ESI+) calcd for $\mathrm{C}_{23} \mathrm{H}_{32} \mathrm{NO}_{2} \mathrm{Si}\left(\mathrm{M}+\mathrm{H}^{+}\right)$382.2197; found 382.2193.

\section{General Procedure II for AgOTf-Catalyzed Cycloisomerization}

To a stirred solution of $\mathbf{3 a - 3 f}$ (1 equiv.) in DMSO (0.06 M) was added AgOTf ( 0.2 equiv.) at ambient temperature. After stirring for $2-4 \mathrm{~h}$ at $120^{\circ} \mathrm{C}$, the reaction mixture was cooled to ambient temperature, quenched with water and extracted with EtOAc. The combined organic layer was washed with brine, dried over $\mathrm{MgSO}_{4}$, and concentrated in vacuo. The residue was purified by flash column chromatography (EtOAc $n$-Hexane $=1: 3$ ) to give title compounds (4a-4f).

\section{7-Phenyl-5H-pyrano[4,3-b]pyridin-5-one (4a)}

Yellow solid of $4 \mathbf{a}(13 \mathrm{mg}, 66 \%)$ was obtained via General Procedure II from $20 \mathrm{mg}$ of $\mathbf{3 a}$.

m.p.: $135-136^{\circ} \mathrm{C} ; \mathrm{R}_{\mathrm{f}}=0.47($ EtOAc $/ n$-Hexane $=1: 1) ;{ }^{1} \mathrm{H}-$ $\operatorname{NMR}\left(800 \mathrm{MHz}, \mathrm{CDCl}_{3}\right) \delta 8.94(\mathrm{dd}, 1 \mathrm{H}, J=1.8,4.6 \mathrm{~Hz}, \mathrm{Ar}-\mathrm{H})$, $8.55(\mathrm{dd}, 1 \mathrm{H}, J=1.4,7.8 \mathrm{~Hz}, \mathrm{Ar}-\mathrm{H}), 7.93-7.91$ (m, 2H, Ar-H), 7.51-7.47 (m, 3H, Ar-H), 7.43 (dd, $1 \mathrm{H}, J=4.6,7.9 \mathrm{~Hz}, \mathrm{Ar}-\mathrm{H})$, 7.23 (s, $1 \mathrm{H}$, vinyl $\mathrm{H}$ ); ${ }^{13} \mathrm{C}-\mathrm{NMR}\left(125 \mathrm{MHz}, \mathrm{CDCl}_{3}\right) \delta 162.1$, 
$157.6,156.3,155.1,137.9,131.5,131.0,129.2,125.9,123.0,117.2$, 103.6; IR (thin film, neat) $v_{\max } 3,067,1,735,1,633,1,561,1,450$, $1,216,1,044,1,035,753,688 \mathrm{~cm}^{-1}$; LR-MS (ESI+) $\mathrm{m} / z 224(\mathrm{M}+$ $\left.\mathrm{H}^{+}\right)$; HR-MS (ESI+) calcd for $\mathrm{C}_{14} \mathrm{H}_{10} \mathrm{NO}_{2}\left(\mathrm{M}+\mathrm{H}^{+}\right)$224.0706; found 224.0707.

\section{4-Methyl-7-phenyl-5H-pyrano[4,3-b]pyridin-5- one (4b)}

Yellow solid of $\mathbf{4 b}(16 \mathrm{mg}, 65 \%)$ was obtained via General Procedure II from $25 \mathrm{mg}$ of $\mathbf{3 b}$.

m.p.: $113-114^{\circ} \mathrm{C} ; \mathrm{R}_{\mathrm{f}}=0.58$, (EtOAc $/ n$-Hexane $\left.=1: 1\right) ;{ }^{1} \mathrm{H}$ NMR (500 MHz, $\left.\mathrm{CDCl}_{3}\right) \delta 8.68(\mathrm{~d}, 1 \mathrm{H}, J=4.9 \mathrm{~Hz}, \mathrm{Ar}-\mathrm{H}), 7.93-$ 7.91 (m, 2H, Ar-H), 7.51-7.47 (m, 3H, Ar-H), 7.21-7.20 (m, $2 \mathrm{H}, \mathrm{Ar}-\mathrm{H}$ \& vinyl $\mathrm{H}), 2.86\left(\mathrm{~s}, 3 \mathrm{H}, \mathrm{CH}_{3}\right) ;{ }^{13} \mathrm{C}-\mathrm{NMR}(125 \mathrm{MHz}$, $\left.\mathrm{CDCl}_{3}\right) \delta 161.3,157.1,156.2,154.6,153.6,131.4,130.9,129.1$, $125.8,125.7,116.2,103.8,22.7$; IR (thin film, neat) $v_{\max } 3,060$, $1,734,1,637,1,574,1,465,1,228,1,058,1,034,847,762,681 \mathrm{~cm}^{-1}$; LR-MS (ESI+) $m / z 238\left(\mathrm{M}+\mathrm{H}^{+}\right)$; HR-MS (ESI+) calcd for $\mathrm{C}_{15} \mathrm{H}_{12} \mathrm{NO}_{2}\left(\mathrm{M}+\mathrm{H}^{+}\right)$238.0863; found 238.0860.

\section{2-Methyl-7-phenyl-5H-pyrano[4,3-b]pyridin-5- one (4c)}

Yellow solid of $4 \mathrm{c}(11 \mathrm{mg}, 54 \%)$ was obtained via General Procedure II from $20 \mathrm{mg}$ of $3 \mathbf{c}$.

m.p.: $129-130^{\circ} \mathrm{C} ; \mathrm{R}_{\mathrm{f}}=0.53($ EtOAc $/ n-$ Hexane $=1: 1) ;{ }^{1} \mathrm{H}-$ NMR $\left(500 \mathrm{MHz}, \mathrm{CDCl}_{3}\right) \delta 8.43(\mathrm{~d}, 1 \mathrm{H}, J=8.1 \mathrm{~Hz}, \mathrm{Ar}-\mathrm{H}), 7.93-$ $7.90(\mathrm{~m}, 2 \mathrm{H}, \mathrm{Ar}-\mathrm{H}), 7.51-7.47$ (m, 3H, Ar-H), 7.30 (d, 1H, J $=8.1 \mathrm{~Hz}, \mathrm{Ar}-\mathrm{H}), 7.22(\mathrm{~s}, 1 \mathrm{H}$, vinyl $\mathrm{H}), 2.72\left(\mathrm{~s}, 3 \mathrm{H}, \mathrm{CH}_{3}\right) ;{ }^{13} \mathrm{C}-$ NMR $\left(125 \mathrm{MHz}, \mathrm{CDCl}_{3}\right) \delta 166.6,162.2,157.6,154.8,137.8$, $131.6,130.9,129.1,125.8,123.3,114.7,103.6,25.5$; IR (thin film, neat) $v_{\max } 3,061,1,752,1,630,1,570,1,460,1,241,1,119$, $1,064,1,030,762,687 \mathrm{~cm}^{-1}$; LR-MS (ESI+) $\mathrm{m} / z 238(\mathrm{M}+$ $\mathrm{H}^{+}$); HR-MS (ESI+) calcd for $\mathrm{C}_{15} \mathrm{H}_{12} \mathrm{NO}_{2}\left(\mathrm{M}+\mathrm{H}^{+}\right)$238.0863; found 238.0857 .

\section{4,7-Diphenyl-5H-pyrano[4,3-b]pyridin-5-one (4d)}

Yellow solid of $\mathbf{4 d}(12 \mathrm{mg}, 60 \%)$ was obtained via General Procedure II from $20 \mathrm{mg}$ of $\mathbf{3 d}$.

m.p.: $142-144^{\circ} \mathrm{C} ; \mathrm{R}_{\mathrm{f}}=0.61($ EtOAc $/ n$-Hexane $=1: 1) ;{ }^{1} \mathrm{H}-$ NMR $\left(500 \mathrm{MHz}^{\mathrm{CDCl}} 3\right.$ ) $\delta 8.86(\mathrm{~d}, 1 \mathrm{H}, J=4.9 \mathrm{~Hz}, \mathrm{Ar}-\mathrm{H}), 7.94-$ 7.92 (m, 2H, Ar-H), 7.50-7.48 (m, 6H, Ar-H), 7.40-7.37 (m, $3 \mathrm{H}, \mathrm{Ar}-\mathrm{H}), 7.28(\mathrm{~d}, 1 \mathrm{H}, J=4.2 \mathrm{~Hz}$, vinyl $\mathrm{H}) ;{ }^{13} \mathrm{C}-\mathrm{NMR}(125$ $\left.\mathrm{MHz}, \mathrm{CDCl}_{3}\right) \delta 160.1,157.5,156.4,154.7,154.6,138.5,131.3$, $131.0,129.1,128.8,128.3,128.2,125.8,125.5,114.3,103.7$; IR (thin film, neat) $v_{\max } 3,058,1,745,1,637,1,568,1,463,1,222$, $1,060,1,029,856,764,696 \mathrm{~cm}^{-1}$; LR-MS (ESI+) $\mathrm{m} / z 300(\mathrm{M}+$ $\left.\mathrm{H}^{+}\right)$; HR-MS (ESI+) calcd for $\mathrm{C}_{20} \mathrm{H}_{14} \mathrm{NO}_{2}\left(\mathrm{M}+\mathrm{H}^{+}\right) 300.1019$; found 300.1017 .

\section{7-phenyl-4-(o-tolyl)-5H-pyrano[4,3-b]pyridin-5- one (4e)}

Yellow solid of 4 e (34 mg, 50\%) was obtained via General Procedure II from $68 \mathrm{mg}$ of $3 \mathrm{e}$.

m.p.: $137-139^{\circ} \mathrm{C} ; \mathrm{R}_{\mathrm{f}}=0.62($ EtOAc/n-Hexane $=1: 1) ;{ }^{1} \mathrm{H}-$ NMR $\left(800 \mathrm{MHz}, \mathrm{CDCl}_{3}\right) \delta 8.90(\mathrm{~s}, 1 \mathrm{H}, \mathrm{Ar}-\mathrm{H}), 7.91-7.88(\mathrm{~m}, 2 \mathrm{H}$, Ar-H), 7.49-7.45 (m, 3H, Ar-H), 7.36-7.32 (m, 2H, Ar-H), 7.307.25 (m, 2H, Ar-H), 7.22-7.18 (m, 1H, Ar-H), $7.06(\mathrm{~d}, J=6.9 \mathrm{~Hz}$,
$1 \mathrm{H}$, vinyl $\mathrm{H}$ ), 2.09 (s, 3H, $\left.\mathrm{CH}_{3}\right) ;{ }^{13} \mathrm{C}-\mathrm{NMR}\left(200 \mathrm{MHz}, \mathrm{CDCl}_{3}\right) \delta$ $159.7,157.9,156.0,154.9,154.4,138.5,134.7,131.2,131.1,123.0$, $129.2,128.6,127.3,125.9,125.8,125.3,115.5,103.3,20.0$; IR (thin film, neat) $v_{\max } 3,066,1,746,1,636,1,568,1,495,1,223,1,027,761$, $688 \mathrm{~cm}^{-1}$; LR-MS (ESI+) $\mathrm{m} / z 314\left(\mathrm{M}+\mathrm{H}^{+}\right)$; HR-MS (ESI+) calcd for $\mathrm{C}_{21} \mathrm{H}_{16} \mathrm{NO}_{2}\left(\mathrm{M}+\mathrm{H}^{+}\right)$314.1176; found 314.1174.

\section{4-(3-nitrophenyl)-7-phenyl-5H-pyrano[4,3-b]pyridin- 5-one (4f)}

$N$-pyranonyl propargylamine $\mathbf{3 f}$ was obtained via General Procedure I from $100 \mathrm{mg}$ of $\mathbf{2}$. The crude $\mathbf{3 f}$ was used for the next step without further purification. Yellow solid of $\mathbf{4 f}$ ( $32 \mathrm{mg}$, $32 \%$ for 2 steps) was obtained via General Procedure II from the crude $\mathbf{3 f}$.

m.p.: $261-262^{\circ} \mathrm{C} ; \mathrm{R}_{\mathrm{f}}=0.48($ EtOAc $/ n$-Hexane $=1: 1) ;{ }^{1} \mathrm{H}-$ NMR $\left(800 \mathrm{MHz}, \mathrm{DMSO}_{-} \mathrm{d}_{6}\right) \delta 9.02(\mathrm{~d}, J=4.8 \mathrm{~Hz}, 1 \mathrm{H}, \mathrm{Ar}-\mathrm{H})$, $8.38(\mathrm{t}, J=1.9 \mathrm{~Hz}, 1 \mathrm{H}$, Ar- $\mathrm{H}$ ), 8.32 (ddd, $J=8.3,2.3,0.9 \mathrm{~Hz}$, $1 \mathrm{H}$, Ar-H), 8.01 (dd, $J=8.1,1.5 \mathrm{~Hz}, 2 \mathrm{H}$, Ar-H), 7.95 (ddd, $J$ $=7.6,1.4,1.1 \mathrm{~Hz}, 1 \mathrm{H}, \operatorname{Ar}-\mathrm{H}), 7.77(\mathrm{t}, J=7.9 \mathrm{~Hz}, 1 \mathrm{H}, \mathrm{Ar}-\mathrm{H})$, 7.60 (s, 1H, Ar-H), 7.58-7.53 (m, 3H, Ar-H), 7.47 (d, $J=4.8 \mathrm{~Hz}$, $1 \mathrm{H}$, vinyl $\mathrm{H}) ;{ }^{13} \mathrm{C}-\mathrm{NMR}\left(200 \mathrm{MHz}, \mathrm{DMSO}-\mathrm{d}_{6}\right) \delta 159.9,156.1$, $155.8,155.6,149.9,147.3,140.2,135.1,130.9,130.8,129.4,129.2$, $125.4,125.3,123.5,123.1,114.0,103.9$; IR (thin film, neat) $v_{\max }$ 3,115, 1,796, 1,637, 1,542, 1,446, 1,219, 1,038, 856, 759, $676 \mathrm{~cm}^{-1}$; LR-MS (ESI+) $m / z 345\left(\mathrm{M}+\mathrm{H}^{+}\right)$; HR-MS (ESI+) calcd for $\mathrm{C}_{20} \mathrm{H}_{13} \mathrm{~N}_{2} \mathrm{O}_{4}\left(\mathrm{M}+\mathrm{H}^{+}\right)$345.0870; found 345.0867.

\section{RESULTS AND DISCUSSIONS}

Our research on the development of a novel synthetic route toward 3-aryl-5-azaisocoumarin commenced with the preparation of cycloisomerization precursors. As shown in Scheme 1, $N$-pyranonyl propargylamine precursors (3a-3k) were prepared according to literature procedures with slight modifications (Dong et al., 2011). The known hydroxy-2-pyrone 1 (Prasad et al., 1995) was treated with tosyl chloride and triethylamine to afford tosylate 2 in $92 \%$ yield. Nucleophilic aromatic substitution of pyrone 2 with commercially available hydrochloride salts of propargylamine or diverse known substituted propargylamine (Experimental details: see Supplementary Materials) readily afforded the corresponding $N$-pyranonyl propargylamines $\mathbf{3} \mathbf{a}-\mathbf{3 h}$ in moderate yields (48-74\%). However, 3-bromopropargyland 1-phenylpropargyl analogs $(\mathbf{3} \mathbf{j}-\mathbf{3 k})$ were not obtained. The nitrophenyl substituted analog $\mathbf{3 i}$ was used for the next reaction without further purification due to inseparable impurities.

With the cycloisomerization precursors $\mathbf{3 a} \mathbf{a} \mathbf{3 i}$ in hand, the cycloisomerization conditions were examined using the simple 3a a model substrate (Table 1). Initially, cycloisomerization of $3 \mathbf{a}$ was conducted under thermal rearrangement conditions. When 3a was heated at $120^{\circ} \mathrm{C}$ in DMSO for $10 \mathrm{~h}$, the desired 5 -azaisocoumarin $\mathbf{4 a}$ was obtained in poor yield (15\%, Table 1, entry 1). The reaction was performed at higher temperature $\left(220^{\circ} \mathrm{C}\right.$ ) in triethylene glycol (TEG) (Han, 2017), affording 5 -azaisocoumarin $\mathbf{4 a}$ in a slightly higher yield (28\%, entry 2 ). The structure of $\mathbf{4 a}$ was confirmed by comparison of its NMR spectrum with previously reported data (Table S1) (Hellal 


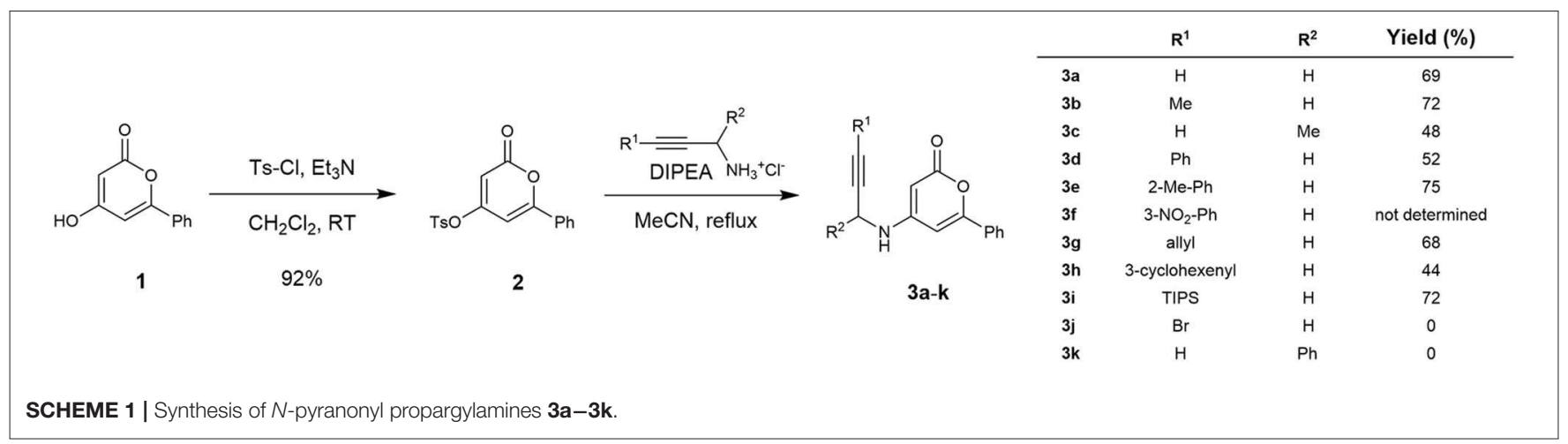

et al., 2008). Recently, many research groups have continuously reported the efficiency of various transition-metal Lewis acid catalysts for alkyne activation in cycloisomerization reaction (Abbiati et al., 2003; Yan et al., 2007; Cacchi et al., 2008; Saito et al., 2009; Jiang et al., 2010; Fei et al., 2011; Mikušek et al., 2016; Vessally et al., 2016; Nizami and Hua, 2017; Sakthivel et al., 2017; Gianni et al., 2018; Lyubov'N et al., 2018; Mancuso et al., 2018; Ahn et al., 2019). To increase the product yield, cycloisomerization was performed in the presence of various metal catalysts, including $\mathrm{Ag}(\mathrm{I}), \mathrm{Au}(\mathrm{I}), \mathrm{Cu}(\mathrm{I}), \mathrm{Cu}(\mathrm{II})$, and In(III). Based on our previous work (Ahn et al., 2019), the cycloisomerization of $\mathbf{3 a}$ was performed with 0.2 equiv. of $\mathrm{AgSbF}_{6}$ (entry 3), affording 4a in a higher yield (38\%) than that without the catalyst after heating in DMSO at $120^{\circ} \mathrm{C}$ for $7 \mathrm{~h}$. Encouraged by this yield improvement with the $\mathrm{Ag}(\mathrm{I})$ catalyst, various counter-anions was screened (entries 4-7) given that the alkyne-activating Lewis acidity of metal ions such as $\mathrm{Ag}(\mathrm{I})$ depends on the nature of the counter-anion (Yamamoto, 2000; $\mathrm{Lu}$ et al., 2017). When $\mathrm{Ag}_{2} \mathrm{O}$ was employed, the yield of $\mathbf{4 a}$ did not notably improve over that obtained with $\mathrm{AgSbF}_{6}$ (38\%, entry 4). However, the yields increased slightly when $\mathrm{AgCl}(50 \%$, entry 5) or $\mathrm{AgNO}_{3}$ (53\%, entry 6) were used. The best result was obtained when the cycloisomerization was performed with AgOTf, furnishing $4 a$ in $66 \%$ yield (entry 7). Moreover, the reaction proceeded to completion much faster $(2 \mathrm{~h})$ than with $\mathrm{AgSbF}_{6}$. Although regioisomeric byproducts were not isolated as expected, the optimal yield for the cycloisomerization of $N$ pyranonyl propargylamine precursors $\mathbf{3 a}$ was slightly lower than that reported in a previous study on pyridocoumarins (Ahn et al., 2019), likely because of the greater degree of decomposition of the acid-labile pyrone moiety. When the reaction was conducted with $\mathrm{AuCl}$, the yield was reduced compared to that observed in the thermal aza-Claisen rearrangement in TEG (24\%, entry 8$)$. Furthermore, the desired cycloisomerization product $4 \mathbf{a}$ was not obtained when using $\mathrm{InCl}_{3}$ (entry 9). $\mathrm{Cu}(\mathrm{I})$ and $\mathrm{Cu}(\mathrm{II})$ catalysts afforded 5-azaisocoumarin $\mathbf{4 a}$ in better yields than with $\mathrm{Au}(\mathrm{I})$ or In(III) catalysts (33\%-62\%; entries $10-14)$. Although CuI afforded $4 \mathbf{a}$ in the best yield among the copper catalysts tested herein (62\%, entry 11$)$, the yield of $\mathbf{4 a}$ and reaction rate did not show any notable improvements over that obtained with AgOTf. Other polar solvents including DMF (entry 15), MeCN (entry 16), and ionic liquid systems (entries 17-18), resulted in lower
TABLE 1 | Investigation of reaction conditions for the cycloisomerization of $\mathbf{3 a}$.

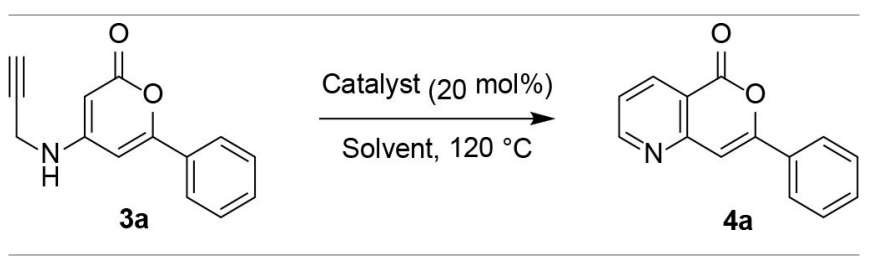

\begin{tabular}{|c|c|c|c|c|}
\hline Entry & Catalyst & Solvent $^{\mathrm{a}}$ & Time (h) & Yield (\%) \\
\hline 1 & - & DMSO & 10 & 15 \\
\hline 2 & - & TEG ${ }^{b}$ & 3 & 28 \\
\hline 3 & $\mathrm{AgSbF}_{6}$ & DMSO & 7 & 38 \\
\hline 4 & $\mathrm{Ag}_{2} \mathrm{O}$ & DMSO & 5 & 38 \\
\hline 5 & $\mathrm{AgCl}$ & DMSO & 4 & 50 \\
\hline 6 & $\mathrm{AgNO}_{3}$ & DMSO & 2 & 53 \\
\hline 7 & AgOTf & DMSO & 2 & 66 \\
\hline 8 & $\mathrm{AuCl}$ & DMSO & 3 & 24 \\
\hline 9 & $\mathrm{InCl}_{3}$ & DMSO & 24 & $N R^{c}$ \\
\hline 10 & $\mathrm{Cu}_{2} \mathrm{O}$ & DMSO & 4 & 61 \\
\hline 11 & Cul & DMSO & 2 & 62 \\
\hline 12 & $\mathrm{CuCl}$ & DMSO & 6 & 58 \\
\hline 13 & $\mathrm{CuBr}$ & DMSO & 4 & 33 \\
\hline 14 & $\mathrm{Cu}(\mathrm{OAc})_{2}$ & DMSO & 4 & 46 \\
\hline 15 & AgOTf & DMF & 5 & 46 \\
\hline 16 & AgOTf & $\mathrm{MeCN}^{\mathrm{d}}$ & 21 & 52 \\
\hline 17 & AgOTf & $\mathrm{DMSO} / \mathrm{L}^{\mathrm{e}}$ & 28 & 25 \\
\hline 18 & AgOTf & $\mathrm{IL}^{f}$ & 28 & $\mathrm{NR}^{\mathrm{c}}$ \\
\hline
\end{tabular}

${ }^{a} 0.06 \mathrm{M}$ solution of $\mathbf{3 a} ;{ }^{b} 0.05 \mathrm{M}$ solution of $\mathbf{3} \boldsymbol{a}$ in triethylene glycol at $220-230^{\circ} \mathrm{C}$.

${ }^{c}$ Starting material was recovered; ${ }^{d}$ reflux.

${ }^{e, f} / \mathrm{L}=$ lonic liquid $=\left[\mathrm{EMIm}_{[}\right]\left[\mathrm{EtSO}_{4}\right], \mathrm{DMSO}:[\mathrm{EMIm}]\left[\mathrm{EtSO}_{4}\right]=10: 1$.

reaction rates and yields of $\mathbf{4 a}$. In particular, the use of an ionic liquid hampered the cycloisomerization, contrary to previous reports (Corma et al., 2005; Neatu et al., 2009; Mancuso et al., 2019). The lower yields obtained in ionic liquids was attributed to the inhibition of the interaction between the alkyne and metal catalyst by the highly polar solvent.

Next, the scope and limitations of the newly developed $\mathrm{Ag}(\mathrm{I})$-catalyzed 3-aryl-5-azaisocoumarin synthesis were 
TABLE 2 | Substrate scope of the AgOTf-catalyzed cycloisomerization toward 3-aryl-5-azaisocoumarin.
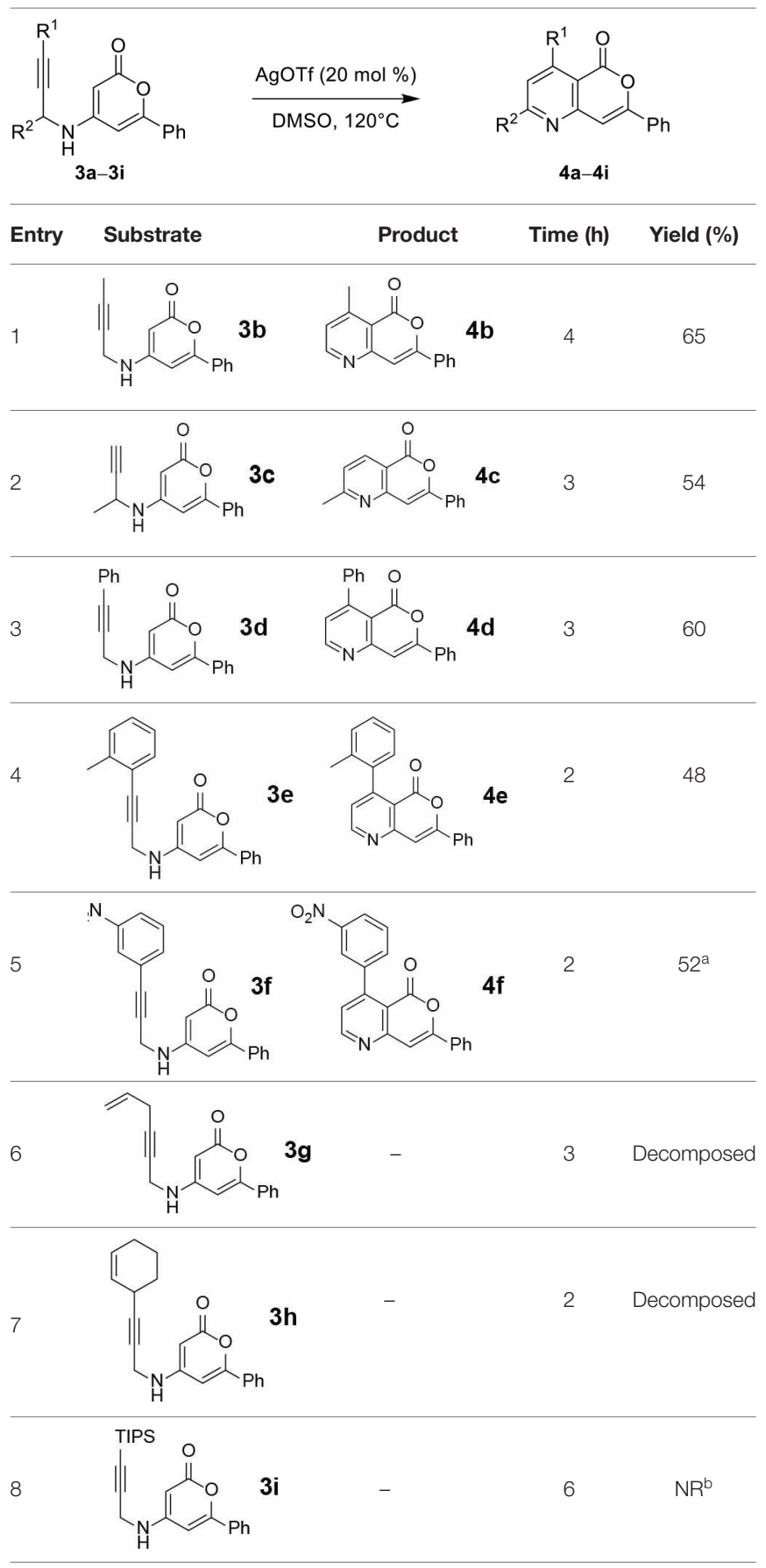

a Two steps yield from 2 ; ${ }^{b}$ Starting material was recovered.

examined (Table 2). We attempted to synthesize the 3-aryl5 -azaisocoumarins from corresponding substituted alkynes (3b-3i). Methyl (3b-3c; entry 1-2) and phenyl substituted (3d3f; entry 3-5) propargylamines generated the corresponding 3-aryl-5-azaisocoumarins (4b-4f) without any notable yield decreases. However, cycloisomerization under standard condition of enynylamine precursors (3g-3h; entry 6-7) resulted in an unidentifiable mixture of byproducts, whereas only substrates were recovered when the reactions were conducted at ambient temperature. These results were attributed to preference of the metal-alkyne complex for reaction with the electron-rich alkene rather than enolizable $\alpha$-position. Although the cycloisomerization reaction of triisopropylsilyl (TIPS) propargylamine (3i) was allowed to proceed for $24 \mathrm{~h}$, only substrate $3 \mathbf{i}$ was recovered in $88 \%$ yield (entry 8 ). This implied that the bulky silyl substituents of alkyne can interfere with formation of the metal-alkyne complex.

\section{CONCLUSION}

In conclusion, an alternative synthetic route for 3-aryl-5azaisocoumarins was developed which includes novel approach to install pyridine moieties on a readily available phenyl-2-pyrone precursor via regioselective metal-catalyzed cycloisomerization. Through an intensive preliminary investigation, AgOTf was determined to be an adequate alkyne-activation catalyst and was applicable in rearrangement of $\mathrm{N}$-pyranonyl propargylamines bearing methyl or phenyl substituents to C-6 or C-8 substituted 3-aryl-5-azaisocoumarin. Derivatization of the pyridine moiety was accomplished efficiently via three-step reaction using the common starting material hydroxy-2-pyrone $\mathbf{1}$ and known propargylamines. Considering that 3-phenyl-5-azaisocoumarin 4a, which was synthesized previously as a regioisomeric mixture using conventional lactonization strategies, was obtained regioselectively in moderate yield, this novel step-economical procedure can be widely utilized in derivatization of C-3, C-6, and C-8 of 5-azaisocoumarins. Further studies on the synthetic applications of the newly developed method are currently underway.

\section{DATA AVAILABILITY STATEMENT}

All datasets generated for this study are included in the article/Supplementary Material.

\section{AUTHOR CONTRIBUTIONS}

YH conceptualized the work. JY and CL synthesized all compound and performed m.p. $\mathrm{R}_{\mathrm{f}}$, NMR, IR, LRMS, and HRMS analysis. The manuscript was written by $\mathrm{CL}$ and $\mathrm{YH}$. All authors approved the manuscript in its final form for publication.

\section{FUNDING}

This research was funded by the National Research Foundation of Korea, NRF-2020R1F1A1058295.

\section{SUPPLEMENTARY MATERIAL}

The Supplementary Material for this article can be found online at: https://www.frontiersin.org/articles/10.3389/fchem. 2020.00772/full\#supplementary-material 


\section{REFERENCES}

Abbiati, G., Arcadi, A., Bianchi, G., Di Giuseppe, S., Marinelli, F., and Rossi, E. (2003). Sequential amination/annulation/aromatization reaction of carbonyl compounds and propargylamine: A new one-pot approach to functionalized pyridines. J. Org. Chem. 68, 6959-6966. doi: 10.1021/jo0347260

Ahn, S., Yoon, J. A., and Han, Y. T. (2019). Total synthesis of the natural pyridocoumarins goniothaline A and B. Synthesis 51, 552-556. doi: 10.1055/s-0037-1610909

Begouin, A., Maria-João, and Queiroz, R. P. (2011). Tandem palladium/charcoalcopper(I) iodide (Pd/C-CuI) catalyzed Sonogashira coupling and intramolecular cyclization from 2-bromonicotinic acid ( $=2$-bromopyridine3 -carboxylic acid) and ethynylarenes to 4-zzaphthalides (=furo[3,4-b]pyridin$5(7 H)$-ones) and 5-azaisocoumarins ( $=5 H$-pyrano[4, 3-b]pyridin-5-ones). Helv. Chim. Acta 94, 1792-1801. doi: 10.1002/hlca.201100060

Cacchi, S., Fabrizi, G., and Filisti, E. (2008). $N$-propargylic $\beta$-enaminones: Common intermediates for the synthesis of polysubstituted pyrroles and pyridines. Org. Lett. 10, 2629-2632. doi: 10.1021/ol800518j

Corma, A., García, H., and Leyva, A. (2005). Palladium catalyzed cycloisomerization of 2,2-diallylmalonates in imidazolium ionic liquids. J. Organomet. Chem. 690, 3529-3534. doi: 10.1016/j.jorganchem.2005.02.044

Dong, Y., Nakagawa-Goto, K., Lai, C.-Y., Morris-Natschke, S. L., Bastow, K. F., and Lee, K.-H. (2011). Antitumor agents 287. Substituted 4-amino- $2 \mathrm{H}$ pyran-2-one (APO) analogs reveal a new scaffold from neo-tanshinlactone with in vitro anticancer activity. Bioorg. Med. Chem. Lett. 21, 2341-2344. doi: 10.1016/j.bmcl.2011.02.084

Fei, N., Yin, H., Wang, S., Wang, H., and Yao, Z.-J. (2011). $\mathrm{CuCl}_{2}$-promoted 6-endo-dig chlorocyclization and oxidative aromatization cascade: Efficient construction of 1-azaanthraquinones from $\mathrm{N}$-propargylaminoquinones. Org. Lett. 13, 4208-4211. doi: 10.1021/ol201542h

Foye, W. O. (2008). Foye's Principles of Medicinal Chemistry. Philadelphia, PA: Lippincott Williams \& Wilkins.

Gaikwad, P. L., Gandhi, P. S., Jagdale, D. M., and Kadam, V. J. (2012). The use of bioisosterism in drug design and molecular modification. Am. J. Pharmtech Res. 2, 1-23. Available online at: http://ajptr.com/assets/upload/publish_article/ AJPTR\%20article\%20Priyanka\%20Gaikwad_9133.pdf

Gianni, J., Pirovano, V., and Abbiati, G. (2018). Silver triflate/p-TSA co-catalysed synthesis of 3-substituted isocoumarins from 2-alkynylbenzoates. Org. Biomol. Chem. 16, 3213-3219. doi: 10.1039/c8ob00436f

Han, Y. T. (2017). Synthesis of proposed structure of rennellianone B: A study on rearrangement of anthraquinonyl propargyl ether toward 2H-pyranoanthraquinone. Tetrahedron Lett. 58, 556-558. doi: 10.1016/j.tetlet.2016.12.080

Hellal, M., Bourguignon, J.-J., and Bihel, F. J.-J. (2008). 6-endo-dig cyclization of heteroarylesters to alkynes promoted by Lewis acid catalyst in the presence of Brønsted acid. Tetrahedron Lett. 49, 62-65. doi: 10.1016/j.tetlet.2007.11.020

Jiang, C., Xu, M., Wang, S., Wang, H., and Yao, Z.-J. (2010). Azaanthraquinone assembly from $\mathrm{N}$-propargylamino quinone via a $\mathrm{Au}(\mathrm{I})$-catalyzed 6-endo-dig cycloisomerization. J. Org. Chem. 75, 4323-4325. doi: 10.1021/jo1006637

Kim, E., Lim, S.-M., Kim, M.-S., Yoo, S.-H., and Kim, Y. (2017). Phyllodulcin, a natural sweetener, regulates obesity-related metabolic changes and fat browning-related genes of subcutaneous white adipose tissue in high-fat dietinduced obese mice. Nutrients 9:1049. doi: 10.3390/nu9101049

Li, J., Chin, E., Lui, A. S., and Chen, L. (2010). One-pot synthesis of phthalides via regioselective intramolecular cyclization from ortho-alkynylbenzaldehydes. Tetrahedron Lett. 51, 5937-5939. doi: 10.1016/j.tetlet.2010.09.023

Lu, Z., Han, J., Okoromoba, O. E., Shimizu, N., Amii, H., Tormena, C. F., et al. (2017). Predicting couterion effects using a gold affinity index and a hydrogen bonding basicity index. Org. Lett. 19, 5848-5851. doi: 10.1021/acs.orglett.7b02829

Lyubov'N., S., Sagitova, E. F., Markova, M. V., Igor,A., U., et al. (2018). Acylethynylpyrroles as a platform for the one-pot access to 2-(pyrrol-2yl)-3-acylpyridines via the dihydrogenative annelation with propargylamine. Tetrahedron Lett. 59, 4047-4049. doi: 10.1016/j.tetlet.2018.09.066

Mancuso, R., Mili,è, R., Palumbo Piccionello, A., Olivieri, D., Della Ca, N., Carfagna, C., et al. (2019). Catalytic carbonylative double cyclization of 2(3-hydroxy-1-yn-1-yl) phenols in ionic liquid leading to furobenzofuranone derivatives. J. Org. Chem. 84, 7303-7311. doi: 10.1021/acs.joc.9b00952
Mancuso, R., Pomelli, C. S., Chiappetta, P., Gioia, K. F., Maner, A., Marino, N., et al. (2018). Divergent syntheses of (Z)-3-alkylideneisobenzofuran-1 $(3 H)$-ones and $1 H$-isochromen-1-ones by copper-catalyzed cycloisomerization of 2-alkynylbenzoic acids in ionic liquids. J. Org. Chem. 83, 6673-6680. doi: 10.1021 acs.joc.8b00923

Matsuda, H., Shimoda, H., Yamahara, J., and Yoshikawa, M. (1998). Immunomodulatory activity of thunberginol a and related compounds isolated from Hydrangeae Dulcis Folium on splenocyte proliferation activated by mitogens. Bioorg. Med. Chem. Lett. 8, 215-220. doi: 10.1016/s0960-894x(97)10221-9

Mikušek, J., Matouš, P., Matoušová, E., Janoušek, M., Kuneš, J., and Pour, M. (2016). Substrate control in the gold (I)-catalyzed cyclization of $\beta$-propargylamino acrylic esters and further transformations of the resultant dihydropyridines. Adv. Synth. Catal. 358, 2912-2922. doi: $10.1002 /$ adsc.201600412

Neatu, F., Pârvulescu, V. I., Michelet, V., Gênet, J.-P., Goguet, A., and Hardacre, C. (2009). Gold imidazolium-based ionic liquids, efficient catalysts for cycloisomerization of $\gamma$-acetylenic carboxylic acids. New J. Chem. 33, 102-106. doi: $10.1039 / \mathrm{B} 812580 \mathrm{E}$

Nizami, T. A., and Hua, R. (2017). Silver-catalyzed chemoselective annulation of propargyl amines with alkynes for access to pyridines and pyrroles. Tetrahedron 73, 6080-6084. doi: 10.1016/j.tet.2017.09.002

Pal, S., Chatare, V., and Pal, M. (2011). Isocoumarin and its derivatives: An overview on their synthesis and applications. Curr. Org. Chem. 15, 782-800. doi: $10.2174 / 138527211794518970$

Panda, B., Bhadra, J., and Sarkar, T. K. (2011). An approach to highly functionalized quinolines and isoquinolines via a gold-catalyzed benzannulation. Synlett 5, 689-693. doi: 10.1055/s-0030-1259555

Park, J. H., Bhilare, S. V., and Youn, S. W. (2011). NHC-catalyzed oxidative cyclization reactions of 2-alkynylbenzaldehydes under aerobic conditions: Synthesis of O-heterocycles. Org. Lett. 13, 2228-2231. doi: 10.1021/ol200481u

Prasad, J. V., Para, K. S., Tummino, P. J., Ferguson, D., McQuade, T. J., Lumney, E. A., et al. (1995). Nonpeptidic potent HIV-1 protease inhibitors:(4-hydroxy6-phenyl-2-oxo- $2 H$-pyran-3-yl) thiomethanes that span $\mathrm{P}_{1}-\mathrm{P}_{2}$ ' subsites in a unique mode of binding. J. Med. Chem. 38, 898-905. doi: 10.1021/jm00006a007

Saddiqa, A., Çakmak, O., and Usman, M. (2017). Isocoumarins and 3, 4dihydroisocoumarins, amazing natural products: A review. Turk. J. Chem. 41, 153-178. doi: 10.3906/kim-1604-66

Saeed, A. (2003). Synthesis of montroumarin. Z. Naturforsch. 58, 691-696. doi: 10.1515/znc-2003-9-1017

Saeed, A. (2016). Isocoumarins, miraculous natural products blessed with diverse pharmacological activities. Eur. J. Med. Chem. 116, 290-317. doi: 10.1016/j.ejmech.2016.03.025

Saito, A., Konishi, T., and Hanzawa, Y. (2009). Synthesis of pyrroles by gold(I)-catalyzed amino-Claisen rearrangement of $\mathrm{N}$-propargyl enaminone derivatives. Org. Lett. 11, 372-374. doi: 10.1021/ol902716n

Sakthivel, S., Sharma, A., and Balamurugan, R. (2017). Silver-catalyzed synthesis of substituted pyridine derivatives from $N$-propargylic $\alpha$-enamino esters. Eur. J. Org. Chem. 3941-3946. doi: 10.1002/ejoc.201700559

Singh, J. B., Mishra, K., Gupta, T., and Singh, R. M. (2018). TBHP-promoted oxidative cyclization of $o$-alkynylquinoline aldehydes: Metal/additive-free domino synthesis of pyrano [4,3-b] quinolin-1-ones. Tetrahedron Lett. 59, 1019-1022. doi: 10.1016/j.tetlet.2018.01.083

Vessally, E., Hosseinian, A., Edjlali, L., Bekhradnia, A., and Esrafili, M. D. (2016). New page to access pyridine derivatives: Synthesis from $\mathrm{N}$-propargylamines. RSC Adv. 6, 71662-71675. doi: 10.1039/C6RA08720E

Yamamoto, H. (2000). Lewis Acids in Organic Synthesis. Weinheim: Wiley-VCH Verlag GmbH.

Yan, J.-Z., Li, J., and Rao, G.-W. (2007). One-pot synthesis of new A-ring fused steroidal pyridines. Steroids 72, 736-739. doi: 10.1016/j.steroids.2007.06.002

Yoon, J. A., and Han, Y. T. (2019). Efficient synthesis of pyrido[3,2-c]coumarins via silver nitrate catalyzed cycloisomerization and application to the first synthesis of polyneomarline C. Synthesis 51, 4611-4618. doi: 10.1055/s-0037-1610730

Yoshikawa, M., Harada, E., Naitoh, Y., Inoue, K., Matsuda, H., Shimoda, H., et al. (1994). Development of bioactive functions in Hydrangeae Dulcis Folium. III. On the antiallergic and antimicrobial principles of Hydrangeae Dulcis Folium. (1). Thunberginols A, B, and F. Chem. Pharm. Bull. 42, 2225-2230. doi: $10.1248 / \mathrm{cpb} .42 .2225$ 
Yoshikawa, M., Matsuda, H., Shimoda, H., Shimada, H., Harada, E., Naitoh, Y., et al. (1996). Development of bioactive functions in Hydrangeae Dulcis Folium. V. On the antiallergic and antimicrobial principles of hydrangeae dulcis folium. (2). Thunberginols $\mathrm{C}, \mathrm{D}$, and $\mathrm{E}$, thunberginol G 3'-O-glucoside, (-)-hydrangenol 4'-O-glucoside, and (+)-hydrangenol 4'-O-glucoside. Chem. Pharm. Bull. 44, 1440-1447. doi: 10.1248/cpb.44.1440

Yoshikawa, M., Uchida, E., Chatani, N., Murakami, N., and Yamahara, J. (1992). Thunberginols A, B, and F, new antiallergic and antimicrobial principles from Hydrangeae Dulcis Folium. Chem. Pharm. Bull. 40, 3121-3123. doi: $10.1248 / \mathrm{cpb} .40 .3121$
Conflict of Interest: The authors declare that the research was conducted in the absence of any commercial or financial relationships that could be construed as a potential conflict of interest.

Copyright $\odot 2020$ Yoon, Lim and Han. This is an open-access article distributed under the terms of the Creative Commons Attribution License (CC BY). The use, distribution or reproduction in other forums is permitted, provided the original author(s) and the copyright owner(s) are credited and that the original publication in this journal is cited, in accordance with accepted academic practice. No use, distribution or reproduction is permitted which does not comply with these terms. 